\title{
Estudo das Propriedades Psicométricas do Teste de Memória de Reconhecimento - TEM-R
}

\author{
Fabián Javier Marín Rueda" \\ Universidade São Francisco, Itatiba, Brasil
}

\begin{abstract}
RESUMO
O objetivo foi verificar as qualidades psicométricas do Teste de Memória de Reconhecimento (TEM-R). Num primeiro momento, uma versão inicial do TEM-R foi aplicada em 137 estudantes universitários. Verificou-se que dos 64 itens iniciais, 15 não apresentaram nenhuma frequência de resposta. Com base nisso, procedeu-se a uma reconfiguração do instrumento, fixando o número de 49 itens. Uma nova aplicação foi realizada, participando 531 estudantes universitários. Os resultados da estrutura interna mostraram uma adequação ao modelo Rasch, a não existência de vieses nos itens por meio da análise do funcionamento diferencial dos itens, e uma estrutura fatorial adequada. Foram observados índices de precisão satisfatórios. Dessa forma, o TEM-R apresentou propriedades psicométricas adequadas para sua utilização na realidade brasileira.
\end{abstract}

Palavras-chave: memória; testes psicológicos; psicometria; validade; precisão.

\begin{abstract}
Study of The Psychometric Properties of The Memory Test of Recognition - TEM-R

The purpose was to verify the psychometric qualities of the Memory Test of Recognition (TEM-R). In a first moment an initial version of the TEM-R was applied at 137 college students. It was found that from the 64 initial items, 15 did not show any frequency response. Based on this it was proceeded a reconfiguration of the instrument, fixing the number of 49 items. It was accomplished a new enforcement where participated 531 college students. The results of the internal structure showed an adequacy to the Rasch model, a absence of bias in the items through the analysis of differential items functioning, and an appropriate factor structure. We observed satisfactory reability indexes. Thus, the TEM-R presented adequate psychometric properties for use in the Brazilian reality.
\end{abstract}

Keywords: memory; psychological tests; psychometry; validity; reability.

Na história do estudo do construto memória, o primeiro investigador experimental foi Ebbinghaus, que, em 1885, se interessou em saber qual a quantidade de informação que as pessoas poderiam se lembrar, imediatamente após a sua apresentação (Tulving \& Craik, 2000). As ideias de Ebbinghaus tiveram influência, ao longo do século XX, nas pesquisas sobre memória humana. Segundo Tulving e Craik (2000), foi a partir de Ebbinghaus que a memória começou a ser testada por lembrança, reconhecimento e reconstrução, assim como por uma variedade de medidas indiretas, sendo que os materiais utilizados para isso também eram variados, assim como também a forma de apresentação, as estratégias que os sujeitos usavam para estudá-las e as expectativas a respeito do teste. O resultado foi uma grande quantidade de informação acumulada sobre como os humanos aprendem em determinadas situações, além da formulação de muitas hipóteses para integrar as diferentes definições e propostas para entender a memória humana.

Especificamente no que se refere à memória de reconhecimento, ela diz respeito a reconhecer que um estímulo visualizado no momento não é novo, ou seja, reconhecer que o mesmo já foi visto anteriormente. $\mathrm{O}$ modelo que sustenta essa definição ficou conhecido como modelo de processo duplo, que supõe que a

Endereço para correspondência: Fabián Javier Marín Rueda - marinfabian@yahoo.com.br. 
memória de reconhecimento está baseada na relembrança e na familiaridade. A relembrança seria um processo mais demorado que a familiaridade, baseado nos conceitos processados e que resulta na recuperação de detalhes contextuais. Por sua vez, a familiaridade seria um processo mais rápido e automático, baseado no processamento das informações perceptivas (Jacoby, 1991; Tulving, 1985).

Dentro desse contexto, muitas vezes é questionado se a relembrança e a familiaridade seriam processos de memória separados e qual seria a relação entre eles, no desempenho em um teste de memória de reconhecimento, uma vez que o reconhecimento poderia ser baseado tanto na familiaridade como na relembrança (Tulving \& Craik, 2000).

Tal relação foi apontada por Kelley e Jacoby (1998), que propuseram que julgamentos de familiaridade seriam atribuições baseadas na fluência perceptual ou conceptual que estaria por trás da primazia. Por sua vez, Tulving (1985) associou familiaridade com repostas de saber e afirmou que elas seriam derivadas da memória semântica e refletiriam a consciência mental. Já para outros estudiosos, como Moscovitch (1992), Moscovitch e Bentin (1993) e Knowlton (1998), o reconhecimento baseado em familiaridade seria funcional e anatomicamente diferente da primazia e da memória semântica; e seria dependente dos processos e mecanismos que apoiam a memória explícita.

Nesse sentido, a literatura especializada tem se preocupado em discutir esses pressupostos. Assim, para Yonelinas, Kroll, Dobbins, Lazzara e Knight (1998), danos no lobo occipital direito e córtex temporal adjacente prejudicariam a primazia perceptual sem afetar o reconhecimento baseado na familiaridade ou na relembrança. Já Wagner e Gabrieli (1998) chegaram à conclusão de que o reconhecimento baseado na familiaridade não seria tipicamente derivado da fluência perceptual, embora a possibilidade de ser derivado da fluência conceptual permaneceria. Autores como Rajaram e Roediger (1997) indicaram que respostas de saber seriam mais influenciadas por manipulações perceptuais do que por manipulações semânticas e sugeriram que elas seriam guiadas pela fluência perceptual. Entretanto, autores como Knowlton (1998) e Yonelinas et al. (1998) afirmaram que, nas suspeitas de amnésia, isso não aconteceria, uma vez que a primazia perceptual estaria intacta, mas ambas as respostas, de lembrar e de saber, no reconhecimento, seriam prejudicadas.
Por sua vez, algumas descobertas sugerem que ambas as respostas, de saber e de lembrar, seriam dependentes do lobo temporal medial e do sistema diencefálico (Parkin \& Walter, 1992; Wheeler, Stuss, \& Tulving, 1997). Também, Blaxton e Theodore (1997) descobriram uma preponderância de respostas de lembrar, em pessoas com lobotomia temporal direita e de respostas de saber, em pessoas com lobotomia temporal esquerda, sugerindo que os lobos temporais direito e esquerdo mediriam respostas de lembrar e de saber, respectivamente. Aggleton e Brown (1999) verificaram que a lembrança seria prejudicada em pacientes com lesões restritas ao hipocampo, mas que a memória de reconhecimento estaria intacta, desde que o dano não se estendesse para o córtex perirrinal e o giro parahipocampal. Uma interpretação desses resultados seria que a lembrança é dependente da formação hipocampal e de suas conexões diencefálicas, enquanto que o saber é mediado pelo córtex perirrinal e suas projeções diencefálicas. Lepage, Habib e Tulving (1998), Schacter e Wagner (1999) e Wagner e Gabrieli (1998) apontaram que existiriam evidências de que regiões posteriores do lobo temporal medial, incluindo o parahipocampo, seriam mais ativadas em testes baseados em familiaridade, enquanto que regiões anteriores seriam mais ativadas em testes exigindo processamento relacional entre itens, que se refeririam à lembrança.

Assim sendo, a suposição de que a relembrança e a familiaridade contribuiriam independentemente com o desempenho em testes de reconhecimento tem sido controversa. Uma alternativa para essa suposição de independência seria que a relembrança e a familiaridade se encontram em uma relação redundante, ou seja, a relembrança não poderia ocorrer, a não ser que a familiaridade ocorresse primeiro. Nesse sentido, uma forma de pensar a diferença entre independência e redundância seria com o exemplo de modelos de lembrança gerar/reconhecer versus modelos de devolução direta. Por exemplo, se for dada uma pista para lembrança com o núcleo de uma palavra estudada, as pessoas poderiam ser instruídas a usar essa pista para gerar a primeira palavra que viesse à mente, e então fazer uma verificação de reconhecimento sobre se o item gerado estava ou não em uma lista estudada anteriormente. Com essa estratégia, o reconhecimento seria um estágio posterior do processamento, que ocorreria apenas se uma palavra estudada tivesse sido gerada e, então, dessa forma, os dois não poderiam ser 
independentes. Assim sendo, a geração de itens estudados estaria "acima" da linha de base, por causa dos efeitos da memória automática ou inconsciente (Jacoby \& Hollingshead, 1990).

Por sua vez, a concepção proposta por Tulving (1985) foi que lembrar e saber seriam estados de percepção que refletiriam a consciência automental e mental e que seriam dois tipos de consciência que caracterizam, respectivamente, sistemas de memória episódica e semântica. Embora Tulving (1985) tenha usado testes de lembrança livre, de lembrança com pista e de reconhecimento, foi a memória de reconhecimento que se tornou o paradigma lembrar/saber mais usado normalmente, uma vez que a memória de reconhecimento seria a mais provável de estar associada com experiências de saber e de lembrar, especialmente quando o reconhecimento fosse acompanhado apenas por sensações de familiaridade. Além disso, como destacou Mandler (1980), os dois estados de consciência capturados por respostas de lembrar e de saber seriam relevantes para as teorias de componente dual da memória de reconhecimento, que sustentam que o reconhecimento poderia ser realizado tanto por um como por dois processos independentes, quais sejam, relembrança e familiaridade.

Quanto à forma de avaliação da memória de reconhecimento, tradicionalmente tem ocorrido por meio de estímulos verbalvisuais, (palavras) ou figuras. Nesse sentido, vários estudos têm procurado investigar e comparar o desempenho em testes que utilizam, ambos, modalidades de apresentação. Dentro desse contexto, Ballesteros, Reales e Manga (1999), Stenberg, Radeborg e Hedman (1995) e Mintzer e Snodgrass (1999) verificaram que o reconhecimento de figuras é sistematicamente maior que o de palavras, em testes que apresentam as duas modalidades de estímulos. Nesses três experimentos, foi evidenciado um melhor desempenho, no caso da figura-figura, ou seja, quando o estimulo estudado foi uma figura e o mesmo foi apresentado, no teste, também como figura. Os casos figura-palavra e palavra-palavra obtiveram o mesmo número de acertos e, por fim, a palavra-figura foram os itens mais difíceis. Dessa forma, os três experimentos mostraram que as figuras são mais facilmente reconhecidas do que as palavras; porém, quando a palavra é apresentada primeiro e, no teste, ela se encontra como figura, o item torna-se o mais difícil.

Em 1971, Paivio (1971) já tinha proposto a teoria da codificação dupla, na qual figuras e palavras seriam estocadas, em sistemas independentes mas relacionados em certo ponto, uma vez que uma figura seria capaz de evocar uma palavra e vice-versa. Ainda, o autor também já tinha sugerido a maior facilidade no reconhecimento de figuras, em detrimento das palavras, uma vez que, para ele, as figuras evocariam tanto os códigos verbais como os códigos pictóricos, sendo que as palavras apenas evocariam códigos verbais.

Por sua vez, Glaser (1992) afirmou que as figuras e as palavras seriam representações simbólicas dos objetos reais que as pessoas conhecem, mas que as figuras seriam símbolos e as palavras não, uma vez que as figuras apresentam semelhanças físicas com os objetos reais. Assim sendo, os processos de reconhecimento, compreensão e denominação das figuras são semelhantes a esses mesmos processos ocorridos com os objetos reais.

Tulving e Craik (2000) também sugeriram algumas formas de avaliar a memória de reconhecimento. Uma delas seria apresentar um ou mais conjuntos de itens, num primeiro momento, e, posteriormente, solicitar aos sujeitos que devolvessem e julgassem algo, sobre o contexto do item da apresentação anterior. Esse julgamento poderia ser se o item foi ou não apresentado anteriormente. Os itens também poderiam ser apresentados em várias listas diferentes, sendo solicitado, posteriormente, que os indivíduos indicassem em qual lista do teste o item foi apresentado. Os itens também poderiam ser apresentados várias vezes, em cada uma das listas do teste, e, depois, poderia ser pedido aos sujeitos que indicassem com qual frequência um item de teste foi apresentado em cada lista. Também poderia ser solicitado que os indivíduos se lembrassem de aspectos da "fonte" - quem disse a palavra, onde ou quando ela foi apresentada, em qual tipo de voz (masculina ou feminina), ou características de sua apresentação visual (tipo de fonte da letra, tamanho, slides coloridos versus preto e branco).

Já no que se refere aos experimentos de memória de reconhecimento, os sujeitos julgam se um item do teste foi ou não apresentado explicitamente em uma lista estudada anteriormente. Um fato básico é que o acesso à memória de um item exigiria que os sujeitos interpretassem (ou "codificassem") o item no teste, da mesma maneira que eles fizeram quando ele foi originalmente estudado. Em outras palavras, os estímulos apresentados durante o teste devem trazer a mesma percepção ou interpretação significativa que ocorreu 
durante o estudo, para que, dessa forma, se faça contato com o traço de memória do evento original. Esse conceito apresentado é o "princípio de especificidade de codificação", proposto por Tulving (1985). Tulving e Craik (2000) afirmaram que esse princípio se aplica bem mais amplamente do que apenas com palavras ou figuras, já que o contexto da apresentação poderia ativar, de alguma forma, diferentes propriedades e associações para milhares de palavras ou figuras que são, de outra forma, não ambíguas.

Ainda têm-se os testes de memória de reconhecimento de livre escolha e os testes de memória de reconhecimento de escolha forçada. Segundo Tulving (1985), em um teste de reconhecimento de livre escolha ("sim-não"), os itens são apresentados isoladamente com uma sequência ordenada aleatoriamente de itens antigos e novos (normalmente, mas não necessariamente, um número igual de cada) e é exigido aos sujeitos que julguem cada item como antigo ("sim") ou novo ("não"). Em um teste de reconhecimento de escolha forçada, cada item previamente estudado ("antigo") é apresentado, agrupado com um pequeno número de itens não apresentados previamente (distratores), ou itens "novos", e é solicitado ao sujeito que escolha quais desses itens são antigos.

A performance em um teste de reconhecimento, tanto de livre escolha como de escolha forçada, irá depender da natureza dos distratores e, em particular, da sua relação com os itens antigos, no que diz respeito à alguma dimensão, como semelhança semântica, ou à sobreposição de características perceptivas. Por exemplo, itens antigos que são pareados com sinônimos como distratores tornam um teste de memória mais difícil do que se fossem pareados com distratores que são semanticamente não relacionados. Por essa razão, no planejamento de experimentos, deve ser tomado cuidado para assegurar que, entre condições, os distratores não sejam diferentes em sua semelhança com itens antigos, uma exigência que pode ser difícil de controlar, se a comparação ocorre entre diferentes tipos de estímulos, tais como palavras versus figuras ou rostos.

Quanto às tarefas de memória de reconhecimento visual, Nelson (1971) propôs o chamado modelo sensorial-semântico. O autor argumentou que os estímulos visuais e pictóricos apresentam três componentes principais, quais sejam, o semântico, o verbal e o fonético. Para Nelson (1971), uma tarefa que necessita de uma codificação visual de palavras e de figuras começaria pelo processamento dos aspectos físicos dos objetos. No caso desse estímulo ser uma figura, o processamento dessas características visuais permitiria o processamento das características semânticas (seu significado). Posteriormente, se poderia aceder às características fonéticas e visuais das palavras escritas que correspondessem às figuras.

No caso da apresentação de um estimulo visualverbal (palavra escrita), após o processamento das características visuais, Nelson (1979) afirmou que os dois processamentos poderiam acontecer, ou seja, as características semânticas e as características fonéticas. Ressalta-se que acessar as características visuais da figura que correspondem à palavra só seria possível após o processamento semântico, ou seja, a pessoa consegue saber qual é a figura que corresponde à palavra lida, após acessar o seu significado.

Esse modelo considera que as codificações das características visuais são lembradas de forma mais fácil, pelo fato de serem mais susceptíveis a interferências de outras codificações. Assim sendo, Nelson (1979) argumentou que as figuras seriam reconhecidas mais facilmente do que as palavras. Nesse sentido, uma vez que as figuras são compostas por características visuais muito particulares, elas são codificadas de forma diferenciada em relação a estímulos mais simples, como, por exemplo, as palavras.

Dessa forma, e como pode ser percebido, a literatura e os modelos que se referem ao construto da memória e, mais especificamente, à memória de reconhecimento, fornecem uma grande bagagem teórica e conceitual para a avaliação da mesma. No Brasil, hoje em dia, existem quatro instrumentos disponíveis para avaliação da memória (Conselho Federal de Psicologia, 2010). Esses instrumentos são o Teste de Memória Visual - TMV (Pasquali, Veiga, Alves, \& Vasconcelos, 2004), a Bateria de Funções Mentais para Motoristas - BFM-2 (Tonglet, 2000), a Bateria Geral de Funções Mentais - BBGFM (Tonglet, 2007) e o Teste Pictórico de Memória - TEPIC-M (Rueda \& Sisto, 2007).

Dentro desse contexto, e seguindo a proposta da Resolução n. 002/2003, de construir testes psicológicos com qualidades psicométricas adequadas para a realidade brasileira, é que surgiu a ideia de construir um teste para a avaliação da memória de reconhecimento. Assim, o objetivo desta pesquisa foi investigar 
as qualidades psicométricas do Teste de Memória de Reconhecimento (TEM-R).

\section{MÉTODO}

Para construção do Teste de Memória de Reconhecimento (TEM-R), num primeiro momento, foram levados em consideração autores que compararam a diferença entre a memória de reconhecimento para figuras e palavras (Mintzer \& Snodgrass, 1999; Schloerscheidt \& Rugg, 2004; Stenberg et al., 1995). Os experimentos realizados por esses autores apresentavam, aos sujeitos, estímulos que podiam aparecer no formato de figuras ou de palavras e, no momento de responder ao teste, os mesmos estímulos anteriormente apresentados podiam novamente se encontrar no formato de figura ou palavra, ou vice-versa, misturados dentre vários estímulos distratores.

Dessa forma, num primeiro momento, o autor da presente pesquisa selecionou 98 estímulos para compor o TEM-R, sendo que, posteriormente, três psicólogos, com formação há mais de cinco anos, selecionaram 64 itens do total. Os psicólogos eram profissionais com conhecimento de psicometria e que trabalhavam na área da psicologia escolar e educacional. Em que pese ao instrumento não ter sido inicialmente desenvolvido com uma amostra de crianças, o objetivo foi que as palavras selecionadas fossem conhecidas por uma população de baixa idade. Dessa forma, com base na sua experiência, os profissionais fizeram a escolha dos estímulos que ficariam no teste. Dos 64 itens escolhidos, 28 foram selecionados para fazer parte dos itens que não deveriam ser marcados (erros, caso fossem assinalados), uma vez que não seriam considerados alvo. Metade desses itens foi apresentada em forma de figuras em preto e branco (abacaxi, alicate, batata, bolsa, desentupidor, escova, grilo, livro, pé, sapato, serrote, trator, trompete e zebra), enquanto os outros 14 foram apresentados no formato de palavras (cama, capacete, chinelo, chupeta, espelho, faca, garrafa, geladeira, nuvem, porta, sabonete, sofá, sol e calça).

Em relação aos itens alvo, os mesmos representaram 36 do total de 64, sendo que eles foram divididos em quatro tipos de estímulos, quais sejam, 1) alvos que eram apresentados em forma de figura em preto e branco e na folha de resposta apareciam também no formato de figura em preto e branco (figura-figura), 2) itens apresentados em forma de figura em preto e branco e que na folha de resposta encontravam-se na forma de palavras (figura-palavra), 3) itens apresentados como palavras e que, posteriormente, deveriam ser assinalados no formato figura em preto e branco (palavra-figura) e, por fim, 4) itens que eram apresentados como palavras e também encontravam-se nesse formato, na folha de resposta (palavra-palavra). A quantidade de itens em cada um desses agrupamentos foi nove, sendo eles:

1) figura-figura: banana, celular, chave, diploma, guarda-chuva, sanduíche, sorvete, tênis e violão.

2) figura-palavra: chapéu, coelho, lâmpada, mão, martelo, melancia, relógio, sino e tesoura.

3) palavra-figura: avião, bicicleta, borboleta, carro, garfo, pássaro, piano, queijo e helicóptero.

4) palavra-palavra: alface, árvore, caneta, colher, hospital, mesa, peixe, semáforo e televisão.

Com o teste inicial configurado, procedeu-se a uma aplicação piloto. Os achados dessa investigação são descritos no Estudo 1.

\section{Estudo 1}

\section{Participantes}

Participaram 137 estudantes universitários do curso de Psicologia de uma instituição particular do interior do estado de São Paulo. A idade variou de 18 a 43 anos $(M=24,56, D P=5,86)$. Em relação ao sexo, 103 $(75,18 \%)$ foram mulheres.

\section{Procedimentos e Resultados}

Após a explicação do objetivo do teste e da instrução do mesmo, a aplicação ocorreu de forma coletiva e em sala de aula. Para tal, primeiramente foi dado um minuto para que os indivíduos memorizassem as palavras e as figuras e, posteriormente, foi solicitado que os mesmos respondessem o instrumento sem limite de tempo. A coleta de dados foi realizada por dois aplicadores em cada sala de aula e os alunos foram orientados a levantarem a mão, quando não se lembrassem de mais itens, momento no qual um dos aplicadores dirigia-se até o sujeito e marcava o tempo na folha de resposta do teste. Foi verificado que o tempo de resposta variou de 47 segundos até 2 minutos e 11 segundos $(M=1,33, D P=0,19)$. 
Do total de 64 itens que compuseram a primeira versão do TEM-R, foi verificado que 15 deles não apresentaram nenhuma frequência de resposta. Deles, 13 se referiam a itens que eram considerados erros, ou seja, aqueles que de fato a pessoa não deveria assinalar, sendo que sete se referiam a erros representados por figuras (batata, bolsa, grilo, livro, sapato, trator e zebra) e seis a erros representados por palavras (cama, capacete, chinelo, espelho, porta e calça). Por fim, os outros dois itens que não apresentaram frequência de resposta foram itens alvo, sendo o item palavra-figura "avião" e o item palavra-palavra "televisão".

Com base nesses achados, optou-se por reconfigurar o instrumento, retirando os itens que não apresentaram nenhuma frequência de resposta. Assim, o TEM-R ficou composto por 49 itens, sendo 15 considerados erros (7 erros apresentados na forma de figura e 8 como palavras) e 34 estímulos alvos. Dos 34 itens alvo, 9 referiam-se a figura-figura, 9 a figura-palavra, 8 a palavra-figura e 8 a palavra-palavra.

Os itens que compuseram cada um dos agrupamentos encontram-se destacados a continuação:

Erros como figura: abacaxi, alicate, desentupidor, escova, pé, serrote e trompete.

Erros como palavra: chupeta, faca, garrafa, geladeira, nuvem, sabonete, sofá e sol.

Estímulo figura-figura: banana, celular, chave, diploma, guarda-chuva, sanduíche, sorvete, tênis e violão.

Estímulo figura-palavra: chapéu, coelho, lâmpada, mão, martelo, melancia, relógio, sino e tesoura.

Estímulo palavra-figura: bicicleta, borboleta, carro, garfo, helicóptero, pássaro, piano e queijo.

Estímulo palavra-palavra: alface, árvore, caneta, colher, hospital, mesa, peixe e semáforo.

Posteriormente, e com base nesses resultados, procedeu-se a um novo estudo. As análises decorrentes dessa investigação são relatadas no Estudo 2.

\section{Estudo 2}

Com o instrumento reformulado, procedeu-se a uma nova coleta de dados. Ressalta-se que, após essa mudança, estabeleceu-se o tempo máximo de um minuto, para responder o instrumento.

\section{Participantes}

Desta vez, participaram 531 estudantes universitários do estado de Sergipe. Deles, 147 (28,9\%) eram homens. Em relação à idade, houve uma variação entre 17 e 53 anos, com uma média de 23,31 $(D P=6,40)$. Ainda, 22 pessoas não apresentaram informações referentes ao sexo e idade. Destaca-se que houve uma concentração de participantes até os 21 anos de idade, representando $57,5 \%$ do total da amostra. Dos 22 aos 30 anos, ficou concentrado $32,2 \%$ da amostra e, a partir dos 31 anos, houve 50 participantes $(10,30 \%)$.

\section{RESUTADOS E DISCUSSÃO}

$\mathrm{Na}$ análise dos resultados, foi investigada a adequação ao modelo Rasch, o funcionamento diferencial dos itens (DIF) e a estrutura interna por meio da análise fatorial. Nesse sentido, para verificar o ajuste do instrumento ao modelo Rasch, foram considerados os 49 itens do teste. Os resultados dessa análise constam da Tabela 1, na qual são oferecidos os valores em relação à dificuldade dos itens, o erro padrão e os valores de infit e outfit. Segundo Prieto e Velasco (2006), as estatísticas do infit e outfit são indicadores do ajuste dos itens e das pessoas ao modelo e referemse às diferenças entre o valor observado e o previsto pelo modelo. O infit indica as situações de discrepância nas quais os valores de habilidade do sujeito se encontram próximos aos valores de dificuldade dos itens. Por sua vez, o outfit descreve situações nas quais pessoas com alta habilidade erram itens de baixa dificuldade ou pessoas com baixa habilidade acertam itens difíceis. 
Tabela 1

Índices Estatísticos do Ajuste dos Itens ao Modelo Rasch

\begin{tabular}{|c|c|c|c|c|}
\hline Item & Dificuldade & Erro & Infit & Outfit \\
\hline Palavra-Figura Pássaro & 3,58 & 0,15 & 1,01 & 1,29 \\
\hline Palavra-Palavra Caneta & 3,38 & 0,14 & 0,96 & 1,14 \\
\hline Palavra-Palavra Mesa & 3,29 & 0,13 & 0,96 & 1,01 \\
\hline Palavra-Figura Bicicleta & 2,92 & 0,12 & 1,10 & 1,23 \\
\hline Palavra-Palavra Peixe & 2,50 & 0,11 & 0,99 & 1,02 \\
\hline Palavra-Palavra Colher & 2,43 & 0,11 & 1,10 & 1,23 \\
\hline Palavra-Figura Borboleta & 2,42 & 0,11 & 1,00 & 1,01 \\
\hline Palavra-Figura Piano & 2,39 & 0,11 & 1,04 & 1,01 \\
\hline Palavra-Figura Carro & 2,37 & 0,11 & 1,08 & 1,15 \\
\hline Palavra-Palavra Árvore & 2,34 & 0,11 & 1,09 & 1,17 \\
\hline Palavra-Figura Queijo & 2,31 & 0,11 & 0,99 & 0,97 \\
\hline Palavra-Figura Garfo & 1,96 & 0,10 & 1,00 & 0,97 \\
\hline Palavra-Palavra Alface & 1,84 & 0,10 & 0,96 & 1,04 \\
\hline Palavra-Palavra Hospital & 1,80 & 0,10 & 0,97 & 0,96 \\
\hline Figura-Palavra Tesoura & 1,64 & 0,10 & 0,96 & 0,98 \\
\hline Figura-Palavra Lâmpada & 1,61 & 0,10 & 0,93 & 0,89 \\
\hline Figura-Palavra Sino & 1,52 & 0,10 & 0,93 & 0,91 \\
\hline Palavra-Figura Helicóptero & 1,45 & 0,10 & 1,12 & 1,17 \\
\hline Figura-Palavra Martelo & 1,34 & 0,10 & 0,97 & 0,97 \\
\hline Palavra-Palavra Semáforo & 1,21 & 0,09 & 1,03 & 1,02 \\
\hline Figura-Palavra Mão & 1,12 & 0,09 & 0,95 & 0,92 \\
\hline Figura-Palavra Chapéu & 1,08 & 0,09 & 0,99 & 0,98 \\
\hline Figura-Palavra Relógio & 1,00 & 0,10 & 0,96 & 0,98 \\
\hline Figura-Palavra Melancia & 0,95 & 0,10 & 0,99 & 0,99 \\
\hline Figura-Figura Guarda-chuva & 0,71 & 0,10 & 0,96 & 0,96 \\
\hline Figura-Figura Tênis & 0,67 & 0,10 & 0,91 & 0,92 \\
\hline Figura-Palavra Coelho & 0,66 & 0,10 & 0,94 & 0,93 \\
\hline Figura-Figura Sanduíche & 0,46 & 0,10 & 1,00 & 0,98 \\
\hline Figura-Figura Banana & 0,40 & 0,10 & 0,98 & 0,96 \\
\hline Figura-Figura Diploma & 0,40 & 0,10 & 0,89 & 0,85 \\
\hline Figura-Figura Sorvete & 0,35 & 0,10 & 0,93 & 0,91 \\
\hline Figura-Figura Celular & 0,13 & 0,10 & 0,95 & 0,98 \\
\hline Figura-Figura Violão & 0,07 & 0,10 & 1,03 & 1,04 \\
\hline Figura-Figura Chave & $-0,20$ & 0,11 & 0,91 & 0,89 \\
\hline Erro Figura Alicate & $-2,10$ & 0,20 & 1,11 & 2,03 \\
\hline Erro Palavra Faca & $-2,36$ & 0,22 & 1,05 & 1,60 \\
\hline Erro Palavra Garrafa & $-2,41$ & 0,23 & 1,05 & 2,53 \\
\hline Erro Figura Pé & $-2,52$ & 0,24 & 1,07 & 1,60 \\
\hline Erro Figura Abacaxi & $-2,93$ & 0,29 & 1,03 & 0,85 \\
\hline Erro Figura Serrote & $-2,93$ & 0,29 & 1,05 & 2,38 \\
\hline Erro Palavra Chupeta & $-3,43$ & 0,36 & 1,02 & 1,23 \\
\hline Erro Palavra Geladeira & $-3,57$ & 0,38 & 1,00 & 0,93 \\
\hline Erro Palavra Sol & $-3,57$ & 0,38 & 0,99 & 3,58 \\
\hline Erro Figura Trompete & $-3,57$ & 0,38 & 1,03 & 1,66 \\
\hline Erro Palavra Sabonete & $-4,14$ & 0,51 & 0,97 & 3,70 \\
\hline Erro Figura Desentupidor & $-4,43$ & 0,58 & 1,02 & 0,87 \\
\hline Erro Figura Escova & $-4,43$ & 0,58 & 1,00 & 1,31 \\
\hline Erro Palavra Nuvem & $-4,84$ & 0,71 & 0,95 & 0,18 \\
\hline Erro Palavra Sofá & $-4,84$ & 0,71 & 1,02 & 2,27 \\
\hline
\end{tabular}


Para a análise do ajuste dos itens, foi considerado o critério do valor $\mathrm{MnSq}$ situado entre 0,70 e 1,30. Isso, pois valores superiores a 1,30 indicam que as pontuações no item foram imprevisíveis, sugerindo que o item não combinaria com os demais, para a definição do continuum da habilidade, ou que existem problemas em sua formulação. Já valores inferiores a 0,70 indicam pouca variabilidade de pontuações no item, ou seja, o padrão de resposta foi previsível.
Na Tabela 1, observa-se que a dificuldade dos itens variou de 3,58 a -4,84. Pelos índices do infit, todos os itens apresentaram ajuste ao modelo, pois se enquadraram nos intervalos 0,70 a 1,30. Em relação ao outfit, nove itens não se ajustaram a esse critério. A representação dos itens em uma escala do mais difícil para o mais fácil encontra-se na Figura 1.
5

4

3

2

1

0 <mais $>$ |<raro>

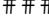

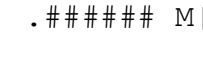

.\#\#\#\# + Fig-Pal Chapéu/Fig-Pal Melancia/Fig-Pal Relógio

.\#\#\#\#\#\#\# ।

.\#\#\#\# | Fig-Pal Coelho/Fig-Fig Guarda-chuva/Fig-Fig Tenis

.\#\#\#\#\#\#｜Ｆig-Fig Sanduíche

.\#\#\#\#\# | Fig-Fig Banana/Fig-Fig Diploma/Fig-Fig Sorvete

.\#\# S| Fig-Fig Celular

\#\#\#\#\# +M Fig-Fig Violão 


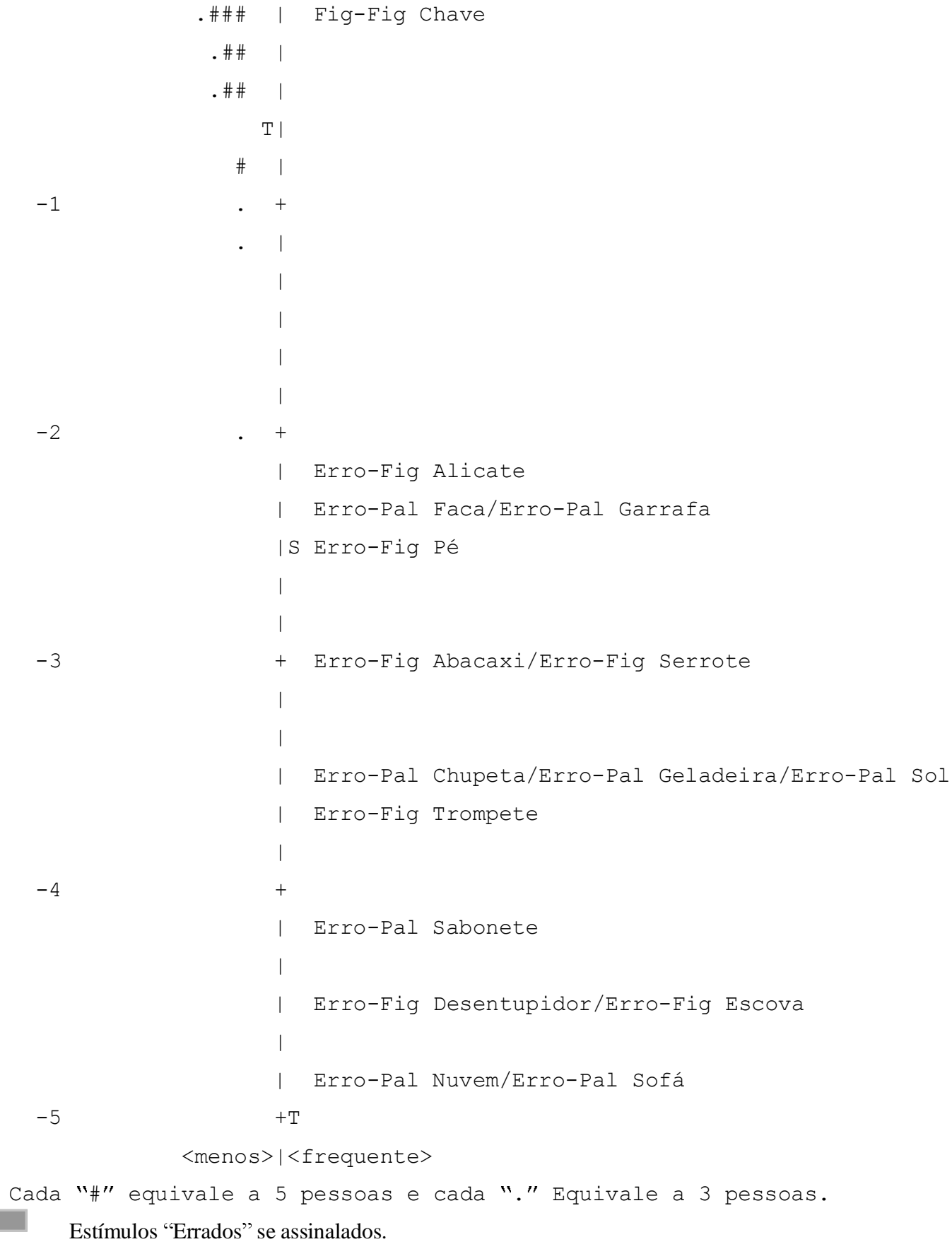

Estímulos alvo apresentados como Figura e que poderiam estar na folha de resposta como Figura ou Palavra.

Estímulos alvo apresentados como Palavra e que poderiam estar na folha de resposta como Palavra ou Figura.

Figura 1. Mapa dos itens por ordem de dificuldade e da distribuição das pessoas.

Pela Figura 1, pode ser observado que a média das pessoas foi superior à média dos itens. Também, desta- ca-se que os itens alvo que eram apresentados inicialmente como palavras e que na folha de resposta do 
teste poderiam estar como palavras ou como figuras foram os itens mais difíceis e menos lembrados. Nesse sentido, os achados vão parcialmente ao encontro do proposto por Mintzer e Snodgrass (1999), Schloerscheidt e Rugg (2004) e Stenberg et al. (1995), que atestaram que os estímulos Palavra-Palavra seriam mais fáceis do que os estímulos Palavra-Figura, que estariam em um patamar intermediário.

Ainda no que se refere à Figura 1, os itens alvo inicialmente mostrados como figuras e que poderiam ser assinalados, caso aparecessem como figuras ou também como palavras, tiveram uma maior pontuação, uma vez que se apresentaram como sendo mais fáceis. Essa informação também encontra-se em certa conso- nância pelo apontado pela literatura, de que os estímulos Figura-Figura seriam os itens mais lembrados, enquanto que as Figura-Palavra seriam mais difíceis. No caso específico desta pesquisa, essa diferenciação pareceria ter acompanhado a proposta da literatura apenas no que tange às Figuras-Figuras. Por fim, os estímulos considerados erros foram os mais fáceis, apresentando, dessa forma, uma menor frequência de respostas.

Quanto aos itens e as pessoas, destaca-se que o erro médio de medida foi de $0,20(D P=0,17)$, para os itens, e de 0,40 $(D P=0,03)$, para as pessoas. Essas informações e outras encontram-se na Tabela 2.

Tabela 2

Parâmetros de Ajuste dos Itens e das Pessoas

\begin{tabular}{lcccccc}
\hline \multirow{2}{*}{ Parâmetros } & \multicolumn{3}{c}{ Itens } & \multicolumn{3}{c}{ Pessoas } \\
\cline { 2 - 6 } & Infit & Outfit & erro & Infit & Outfit & erro \\
\hline Média & 1,00 & 1,25 & 0,20 & 0,99 & 1,13 & 0,40 \\
DP & 0,05 & 0,64 & 0,17 & 0,22 & 1,43 & 0,03 \\
Máximo & 1,12 & 3,70 & 1,02 & 2,18 & 9,90 & 1,21 \\
Mínimo & 0,89 & 0,18 & 0,11 & 0,33 & 0,18 & 0,52 \\
$1,3<>1,5$ & $0(0,00 \%)$ & $1(2,04 \%)$ & & $36(6,83 \%)$ & $23(4,36 \%)$ & \\
$1,5<>2,0$ & $0(0,00 \%)$ & $2(4,08 \%)$ & & $10(1,90 \%)$ & $25(4,74 \%)$ & \\
$<2,0$ & $0(0,00 \%)$ & $6(12,24 \%)$ & & $1(0,19 \%)$ & $47(8,92 \%)$ & \\
\hline
\end{tabular}

A média de infit dos itens foi de $1,00(D P=0,05)$. Assim, é possível interpretar que os itens foram respondidos de acordo com o padrão esperado, que é de 1,00 (Linacre, 2002). Os valores ficaram entre $0,89 \mathrm{e}$ 1,12, ou seja, muito próximos do intervalo $0,70-1,30$. Ainda, não houve itens com valor discrepante. Em relação ao outfit, seu valor médio foi $1,25(D P=0,64)$, sugerindo uma adequação ao modelo. No entanto, tiveram uma variação entre 0,18 e 3,70 . Os itens com valor discrepante foram nove, destacando-se que todos eles eram itens considerados erros.

Sobre as pessoas, no infit observou-se que apenas $11(2,09 \%)$ forneceram escolhas desajustadas, maior que 1,50 (Linacre, 2002). De certa forma, as respostas dessas pessoas não foram compatíveis com o esperado na escala em razão de sua habilidade em memória de reconhecimento. Por sua vez, os dados do outfit registraram $95(17,89 \%)$ pessoas com respostas inesperadas. Dessa forma, os percentuais de desajuste podem ser considerados baixos. Destaca-se que a maioria das pessoas apresentou o padrão esperado, que seria de 1,00 , pois a média do infit foi $0,99(D P=0,22)$ e a do outfit de $1,13(D P=1,43)$, com uma média de erro de $0,40(D P=0,03)$.

Dando continuidade às análises, o estudo referente ao funcionamento diferencial dos itens (DIF) foi realizado no programa Winsteps. A variável de interesse, neste estudo, referiu-se ao sexo das pessoas. Nesse sentido, objetivou-se verificar se os itens do TEM-R possuem o mesmo comportamento estatístico (ou equivalência de medida) quando comparados subgrupos de sujeitos pertencentes à mesma população. Quando essa equivalência não é constatada, significa que há presença de DIF; por sua vez, se essa equivalência é observada, conclui-se que não há DIF. A Tabela 3 fornece os resultados das análises por item, destacando-se que não foram considerados, para análise, aqueles sujeitos que não informaram o sexo, ao responder o teste. 
Tabela 3

Acréscimos de DIF por Sexo, Mudança e Valores de t por Item

\begin{tabular}{|c|c|c|c|c|c|c|}
\hline Sexo & Medida do DIF & Sexo & Medida do DIF & Mudança & $t$ & Item \\
\hline $\mathrm{F}$ & 0,34 & M & 0,56 & $-0,23$ & $-1,04$ & Figura-Figura Banana \\
\hline $\mathrm{F}$ & $-2,96$ & M & $-2,84$ & $-0,12$ & $-0,19$ & Erro Abacaxi \\
\hline $\mathrm{F}$ & 0,74 & M & 0,46 & 0,28 & 1,29 & Figura-Palavra Coelho \\
\hline $\mathrm{F}$ & $-2,65$ & M & $-4,27$ & 1,61 & 1,54 & Erro Serrote \\
\hline $\mathrm{F}$ & 1,52 & M & 1,29 & 0,24 & 1,14 & Palavra-Figura Helicóptero \\
\hline $\mathrm{F}$ & 0,66 & M & 0,82 & $-0,16$ & $-0,75$ & Figura-Figura Guarda-chuva \\
\hline $\mathrm{F}$ & 2,50 & M & 2,50 & $-0,01$ & $-0,02$ & Palavra-Palavra Peixe \\
\hline $\mathrm{F}$ & 1,63 & M & 2,23 & $-0,60$ & $-2,71$ & Palavra-Palavra Hospital \\
\hline $\mathrm{F}$ & $-4,09$ & M & $-2,84$ & $-1,25$ & $-1,61$ & Erro Geladeira \\
\hline $\mathrm{F}$ & 0,37 & M & 0,33 & 0,04 & 0,18 & Figura-Figura Sorvete \\
\hline $\mathrm{F}$ & $-5,20$ & M & $-4,27$ & $-0,94$ & $-0,66$ & Erro Nuvem \\
\hline $\mathrm{F}$ & 2,45 & M & 2,09 & 0,36 & 1,57 & Palavra-Palavra Árvore \\
\hline $\mathrm{F}$ & $-2,10$ & M & $-2,09$ & 0,00 & $-0,01$ & Erro Alicate \\
\hline $\mathrm{F}$ & 1,20 & M & 0,92 & 0,29 & 1,37 & Figura-Palavra Mão \\
\hline $\mathrm{F}$ & 1,20 & M & 1,22 & $-0,02$ & $-0,10$ & Palavra-Palavra Semáforo \\
\hline $\mathrm{F}$ & 2,33 & M & 2,27 & 0,06 & 0,27 & Palavra-Figura Queijo \\
\hline $\mathrm{F}$ & 0,99 & M & 1,04 & $-0,05$ & $-0,25$ & Figura-Palavra Relógio \\
\hline $\mathrm{F}$ & 0,58 & M & 0,88 & $-0,30$ & $-1,42$ & Figura-Figura Tênis \\
\hline $\mathrm{F}$ & 2,03 & M & 1,79 & 0,24 & 1,11 & Palavra-Figura Garfo \\
\hline $\mathrm{F}$ & $-4,09$ & M & $-5,46$ & 1,37 & 0,72 & Erro Desentupidor \\
\hline $\mathrm{F}$ & 1,79 & M & 1,95 & $-0,16$ & $-0,75$ & Palavra-Palavra Alface \\
\hline $\mathrm{F}$ & 0,18 & M & $-0,20$ & 0,38 & 1,62 & Figura-Figura Violão \\
\hline $\mathrm{F}$ & 1,55 & M & 1,76 & $-0,21$ & $-0,97$ & Figura-Palavra Lâmpada \\
\hline $\mathrm{F}$ & $-3,22$ & M & $-4,27$ & 1,05 & 0,97 & Erro Chupeta \\
\hline $\mathrm{F}$ & 0,20 & M & 1,07 & $-0,87$ & $-4,10$ & Figura-Figura Sanduíche \\
\hline $\mathrm{F}$ & 1,47 & M & 1,04 & 0,43 & 2,07 & Figura-Palavra Martelo \\
\hline $\mathrm{F}$ & 3,69 & M & 3,35 & 0,34 & 1,10 & Palavra-Figura Pássaro \\
\hline $\mathrm{F}$ & $-3,38$ & M & $-4,27$ & 0,89 & 0,81 & Erro Sol \\
\hline $\mathrm{F}$ & 2,75 & M & 3,41 & $-0,67$ & $-2,27$ & Palavra-Figura Bicicleta \\
\hline $\mathrm{F}$ & 0,27 & M & 0,73 & $-0,46$ & $-2,13$ & Figura-Figura Diploma \\
\hline $\mathrm{F}$ & $-5,20$ & M & $-4,27$ & $-0,94$ & $-0,66$ & Erro Sofá \\
\hline $\mathrm{F}$ & 2,45 & M & 2,27 & 0,18 & 0,76 & Palavra-Figura Piano \\
\hline $\mathrm{F}$ & $-2,34$ & M & $-2,60$ & 0,26 & 0,49 & Erro Garrafa \\
\hline $\mathrm{F}$ & 1,78 & M & 1,32 & 0,46 & 2,18 & Figura-Palavra Tesoura \\
\hline $\mathrm{F}$ & 3,45 & M & 3,22 & 0,22 & 0,76 & Palavra-Palavra Caneta \\
\hline $\mathrm{F}$ & 2,41 & M & 2,46 & $-0,05$ & $-0,20$ & Palavra-Palavra Colher \\
\hline $\mathrm{F}$ & 3,48 & M & 2,90 & 0,58 & 2,07 & Palavra-Palavra Mesa \\
\hline $\mathrm{F}$ & 0,99 & M & 0,85 & 0,13 & 0,64 & Figura-Palavra Melancia \\
\hline $\mathrm{F}$ & $-3,80$ & M & $-3,14$ & $-0,66$ & $-0,85$ & Erro Trompete \\
\hline $\mathrm{F}$ & 0,08 & M & 0,26 & $-0,18$ & $-0,80$ & Figura-Figura Celular \\
\hline $\mathrm{F}$ & $-2,28$ & M & $-2,60$ & 0,33 & 0,62 & Erro Faca \\
\hline $\mathrm{F}$ & $-4,09$ & M & $-5,46$ & 1,37 & 0,72 & Erro Escova \\
\hline $\mathrm{F}$ & 1,52 & M & 1,50 & 0,02 & 0,10 & Figura-Palavra Sino \\
\hline $\mathrm{F}$ & $-0,31$ & M & 0,07 & $-0,38$ & $-1,64$ & Figura-Figura Chave \\
\hline $\mathrm{F}$ & $-2,57$ & M & $-2,41$ & $-0,16$ & $-0,31$ & Erro Pé \\
\hline $\mathrm{F}$ & 2,51 & M & 2,06 & 0,46 & 2,01 & Palavra-Figura Carro \\
\hline $\mathrm{F}$ & $-4,09$ & M & $-4,27$ & 0,17 & 0,15 & Erro Sabonete \\
\hline $\mathrm{F}$ & 1,09 & M & 1,07 & 0,02 & 0,09 & Figura-Palavra Chapéu \\
\hline $\mathrm{F}$ & 2,32 & M & 2,67 & $-0,35$ & $-1,45$ & Palavra-Figura Borboleta \\
\hline
\end{tabular}


Pode ser observado que dos 49 itens que compõem o TEM-R, apenas dois indicaram diferenciar o sexo (Palavra-Palavra Hospital e Figura-Figura Sanduíche), sendo que ambos favoreceram as mulheres. Levando em consideração que certa quantidade de DIF sempre está presente, julgou-se que não seria necessária a substituição ou retirada dos itens.
Por fim, foi estudada a validade de construto, por meio da análise fatorial. A medida de adequação da amostra de Kaiser-Meyer-Olkin $(\mathrm{KMO}=0,759)$ e o teste de esfericidade de Bartlett $\left(\chi^{2}=3410,80, g l=1176\right.$, $p=0,000)$ indicaram a possibilidade de extração de mais de um fator. Analisando o scree plot (Figura 2), observou-se a possibilidade de três ou quatro fatores.

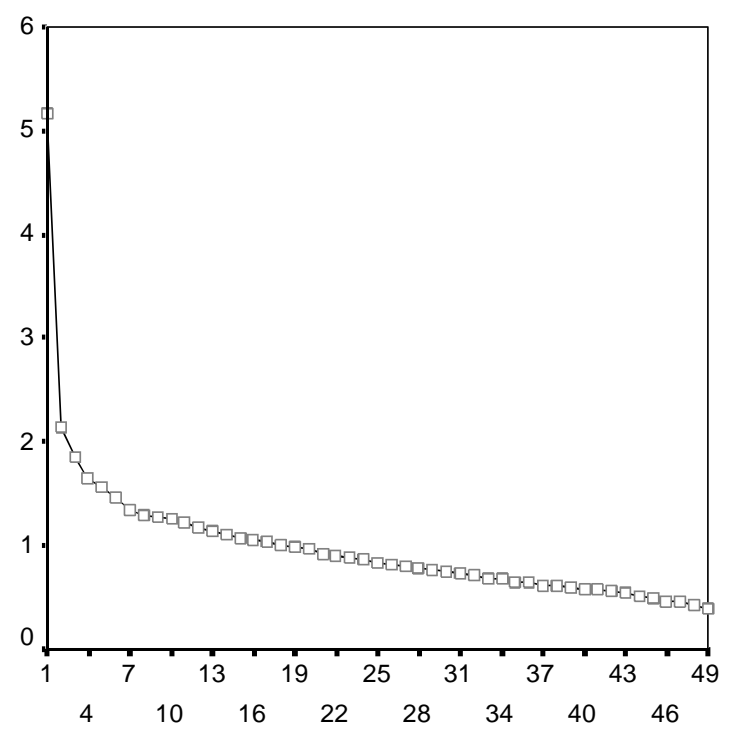

Número de componentes

Figura 2. Gráfico de sedimentação para o TEM-R.

Buscando a melhor solução fatorial, que apresentasse características psicométricas adequadas e que fornecesse uma boa interpretação teórica, após verificar que a estrutura de quatro fatores não se mostrou adequada, optou-se por impor a extração de três fatores. Assim sendo, a estrutura de três fatores se mostrou como sendo a que melhor representou o construto teórico. Todos os itens agruparam-se nos fatores, po- rém, houve cinco itens que apresentaram cargas fatoriais acima de 0,20 (saturação imposta para inclusão do item) e que se agruparam em dois fatores. Os resultados da análise fatorial, carga fatorial dos itens nos fatores, comunalidades, quantidade de variância explicada por fator e seus respectivos eigenvalues são apresentados na Tabela 4. 
Tabela 4

Saturação (Superior a 0,20) por Componentes, com Rotação Quartimax e Normalização de Kaiser, Eigenvaluese e Variância Explicada do TEM-R

\begin{tabular}{|c|c|c|c|c|}
\hline & \multicolumn{4}{|c|}{ Componentes } \\
\hline & 1 & 2 & 3 & h2 \\
\hline Figura-Figura Celular & 0,70 & & & 0,49 \\
\hline Figura-Figura Diploma & 0,69 & & & 0,46 \\
\hline Figura-Figura Guarda-chuva & 0,64 & & & 0,41 \\
\hline Figura-Figura Sanduíche & 0,62 & & & 0,37 \\
\hline Figura-Figura Tênis & 0,61 & & & 0,39 \\
\hline Figura-Figura Banana & 0,60 & & & 0,35 \\
\hline Figura-Figura Sorvete & 0,58 & & & 0,37 \\
\hline Figura-Palavra Lâmpada & 0,56 & & & 0,34 \\
\hline Figura-Figura Chave & 0,55 & & & 0,32 \\
\hline Figura-Palavra Coelho & 0,53 & & $-0,22$ & 0,37 \\
\hline Figura-Palavra Sino & 0,52 & & & 0,31 \\
\hline Figura-Palavra Chapéu & 0,52 & & & 0,30 \\
\hline Figura-Palavra Melancia & 0,50 & & & 0,28 \\
\hline Figura-Palavra Martelo & 0,49 & 0,33 & & 0,30 \\
\hline Figura-Figura Violão & 0,47 & & & 0,25 \\
\hline Figura-Palavra Mão & 0,45 & & & 0,23 \\
\hline Figura-Palavra Relógio & 0,45 & & & 0,24 \\
\hline Figura-Palavra Tesoura & 0,40 & & & 0,22 \\
\hline Palavra-Palavra Alface & & 0,60 & & 0,36 \\
\hline Palavra-Palavra Semáforo & & 0,56 & & 0,34 \\
\hline Palavra-Figura Queijo & & 0,55 & & 0,32 \\
\hline Palavra-Palavra Peixe & & 0,54 & & 0,31 \\
\hline Palavra-Palavra Mesa & & 0,53 & & 0,30 \\
\hline Palavra-Palavra Caneta & & 0,52 & & 0,29 \\
\hline Palavra-Palavra Árvore & & 0,52 & & 0,28 \\
\hline Palavra-Figura Carro & & 0,49 & & 0,26 \\
\hline Palavra-Figura Borboleta & & 0,46 & & 0,29 \\
\hline Palavra-Figura Piano & & 0,45 & & 0,24 \\
\hline Palavra-Figura Pássaro & & 0,45 & & 0,23 \\
\hline Palavra-Palavra Hospital & 0,25 & 0,45 & & 0,30 \\
\hline Palavra-Palavra Colher & & 0,40 & & 0,20 \\
\hline Palavra-Figura Bicicleta & & 0,34 & & 0,19 \\
\hline Palavra-Figura Helicóptero & & 0,34 & & 0,17 \\
\hline Palavra-Figura Garfo & 0,22 & 0,29 & & 0,26 \\
\hline Erro Palavra Sol & & & 0,54 & 0,33 \\
\hline Erro Figura Trompete & & & 0,54 & 0,30 \\
\hline Erro Palavra Faca & & & 0,53 & 0,28 \\
\hline Erro Figura Desentupidor & & & 0,53 & 0,28 \\
\hline Erro Figura Serrote & & & 0,49 & 0,26 \\
\hline Erro Palavra Garrafa & & & 0,48 & 0,24 \\
\hline Erro Figura Abacaxi & & & 0,44 & 0,22 \\
\hline Erro Palavra Nuvem & $-0,31$ & & 0,43 & 0,26 \\
\hline Erro Figura Pé & & & 0,36 & 0,17 \\
\hline Erro Palavra Geladeira & & & 0,31 & 0,18 \\
\hline Erro Palavra Sofá & & & 0,31 & 0,17 \\
\hline Erro Palavra Chupeta & & & 0,29 & 0,09 \\
\hline Erro Figura Escova & & & 0,23 & 0,06 \\
\hline Erro Figura Alicate & & & 0,23 & 0,11 \\
\hline Erro Palavra Sabonete & & & 0,23 & 0,07 \\
\hline Eigenvalues & 5,159 & 2,138 & 1,854 & \\
\hline \% de variância explicada & 20,53 & 8,51 & 7,38 & \\
\hline Total de variância explicada & 36,42 & & & \\
\hline
\end{tabular}


Os 49 itens, distribuídos nos três fatores, explicaram $36,42 \%$ da variância. O primeiro fator apresentou 18 itens e explicou 20,53\% da variância. O mesmo foi constituído por estímulos alvo que foram apresentados no formato de figura e que poderiam aparecer na folha de resposta, no formato tanto de figura quanto de palavra. Destaca-se que dois itens apresentaram cargas fatoriais em mais de um fator, quais sejam, Figura-Palavra Coelho e Figura-Palavra Martelo. Com base na configuração do fator e nas cargas fatoriais desses itens, optou-se por mantê-los no Fator 1, assim como também no teste.

Por sua vez, o segundo fator ficou constituído por 16 itens e explicou $8,51 \%$ de variância. Todos os itens desse fator são estímulos alvo apresentados primeiramente como palavras e que, posteriormente, poderiam ser marcados como palavras ou como figuras. Neste fator, também foi observado que dois itens (Palavra-Palavra Hospital e Palavra-Figura Garfo) apresentaram cargas fatoriais em outros fatores, porém, seguindo o mesmo procedimento adotado anteriormente, os itens foram mantidos.

Finalmente, no terceiro fator, com 15 itens, ficaram agrupados todos os itens considerados erros, explicando 7,38\% da variância. O item Erro Palavra Nuvem também apresentou carga fatorial no Fator 1, sendo a mesma negativa; porém, optou-se por mantê-lo no Fator 3.

Como pode ser observado, a configuração dos itens no teste não seguiu os modelos propostos e esperados segundo as pesquisas de Mintzer e Snodgrass (1999), Schloerscheidt e Rugg (2004) e Stenberg et al. (1995). Porém, a configuraçao apresentada mostrou-se lógica e interpretável, no caso dos itens do TEM-R.

A partir desses resultados, foram calculados os coeficientes de consistência interna de cada fator, por meio do Alfa de Cronbach e do método das duas metades de Spearman-Brown. Os dados de precisão por esses dois métodos indicaram índices satisfatórios para os Fatores 1 e 2, variando de 0,76 a 0,84 . Por sua vez, o Fator 3 apresentou índices de precisão menores $(0,54$, pelo Alfa de Cronbach, e 0,60, pelas duas metades de Spearman-Brown). Ao corrigir o coeficiente de precisão do Fator 3 pela fórmula de Spearman-Brown, o coeficiente aumentou para 0,74 e 0,78 para o Alfa e para as duas metades, respectivamente. Ainda, a precisão para o teste como um todo também foi estudada, sendo obtido coeficientes variando de 0,81 (alfa de Cronbach e duas metades de Guttman) a 0,83 (duas metades de Spearman-Brown). Com base nos coeficientes obtidos, concluiu-se que os índices de precisão foram adequados (Resolução n. 002/2003).

\section{CONSIDERAÇÕES FINAIS}

As análises realizadas na presente pesquisa mostraram-se satisfatórias, tanto no que se refere ao construto teórico da memória de reconhecimento (Mintzer \& Snodgrass, 1999; Schloerscheidt \& Rugg, 2004; Stenberg et al., 1995), quanto aos padrões estabelecidos pela Psicometria (Resolução n. 002/2003). Nesse sentido, se, por um lado, a estrutura interna do TEM-R não se mostrou totalmente em consonância com o proposto pela literatura, os três fatores verificados pela análise fatorial, assim como também as análises realizadas do ajuste ao modelo Rasch e do DIF, permitiram verificar uma estrutura interna do teste analisável e com um padrão psicométrico muito adequado. Ainda, a avaliação da precisão do teste foi considerada satisfatória. Dessa forma, os resultados verificados no TEM-R indicaram que o teste representa uma medida válida e precisa do construto memória de reconhecimento.

Pelos dados, foi verificado que a memória de reconhecimento avaliada pelo TEM-R se dividiria em três fatores, identificados como Figura-Figura e Figura-Palavra (Fator 1), Palavra-Figura e Palavra-Palavra (Fator 2) e Erros (Fator 3). Ainda, o ajuste ao modelo Rasch mostrou-se adequado tanto nos itens como nas pessoas (Linacre, 2002). Por fim, pela análise do DIF, não foram verificados vieses no instrumento no que se refere ao sexo das pessoas.

Com base nas análises realizadas no ajuste ao modelo Rasch, especificamente no "Mapa dos itens" (Figura 2) e na análise fatorial, sugere-se estabelecer um critério de pontuação para o teste. Dessa forma, os estímulos alvo, apresentados como figura e que poderiam ser assinalados como figura ou palavra (Figura-Figura e Figura-Palavra), receberiam 1 (um) ponto, cada vez que assinalados. Já os estímulos apresentados como palavra e que poderiam aparecer como palavra ou figura (itens mais difíceis que os anteriores) passariam a receber 2 (dois) pontos e, por fim, os estímulos considerados Erro (seja no formato de figura ou de palavra) receberiam 3 pontos, caso assinalados pela pessoa. Isso, pois eles são os estímulos que se apresentaram com a menor dificuldade, indicando 
uma baixa frequência de resposta, ou seja, de respostas erradas. Já ao levar em consideração às análises do DIF, pode-se interpretar que a criação de normas específicas para cada sexo não seria necessária.

De forma geral, em que pese aos resultados deste estudo terem mostrado adequadas propriedades psicométricas para o TEM-R, sugere-se que outros estudos sejam realizados, com a finalidade de atender a todas as exigências e recomendações propostas pela Resolução n. 002/2003, visando a excelência no que diz respeito às qualidades psicométricas dos instrumentos de medida utilizados para a realidade brasileira. Nesse sentido, o TEM-R poderia ser considerado um instrumento adequado para a avaliação da memória de reconhecimento em diferentes contextos, como ser na organização, no trânsito, no processo ensino-aprendizagem, desde que sejam realizados estudos com essas populações específicas.

\section{REFERÊNCIAS}

Aggleton, J. P., \& Brown, M. W. (1999). Episodic memory, amnesia and the hippocampal-anterior thalamic axis. Behavioral and Brain Sciences, 22, 425-489.

Ballesteros, S., Reales, J. M., \& Manga, D. (1999). Memoria implícita y memoria explicita intramodal e intermodal: Influencia de las modalidades elegidas y el tipo de estímulos. Psicothema, 11, 831-851.

Blaxton, T. A., \& Theodore, W. H. (1997). The role of the temporal lobes in recognizing visuospatial materials: Remembering versus knowing. Brain and Cognition, 35, 5-25.

Conselho Federal de Psicologia (2010). Sistema de avaliação de testes psicológicos (SATEPSI). Retirado em 11 de janeiro de 2010, de http://www.pol.org.br

Glaser, W. R. (1992). Picture naming. Cognition, 42, 61-105.

Jacoby, L. L. (1991). A process dissociation framework: Separating automatic from intentional uses of memory. Journal of Memory and Cognition, 30, 513-541.

Jacoby, L. L., \& Hollingshead, A. (1990). Toward a generate/recognize model of performance on direct and indirect tests of memory. Journal of Memory, and Language, 29, 433454.

Kelley, C. M., \& Jacoby, L. L. (1998). Subjective reports and process dissociation: Fluency, knowing, and feeling. Acta Psychologica, 98, 127-140.

Knowlton, B. J. (1998). The relationship between remembering and knowing: A cognitive neuroscience perspective. Acta Psychologica, 98, 253-266.

Lepage, M., Habib, R., \& Tulving, E. (1998). Hippocampal PET activations of memory encoding and retrieval: The HIPER model. Hippocampus, 8, 313-322.

Linacre, J. M. (2002). What do Infit and Outfit, Mean-Squared and Standardized mean? Rasch Measurement Transactions, 16,
878. Retirado em 25 de abril de 2006, de http://209.238. 26.90/rmt/rmt82a.htm

Mandler, G. (1980). Recognizing: The judgment of previous occurrence. Psycological Review, 87, 252-271.

Mintzer, M. Z., \& Snodgrass, J. G. (1999). The picture superiority effect: Support for the distinctiveness model. American Journal of Psychology, 112, 113-146.

Moscovitch, M. (1992). Memory and working with memory: A component process model based on modules and central systems. Journal of Cognitive Neuroscience, 4, 257-267.

Moscovitch, M., \& Bentin, S. (1993). The fate of repetition effects when recognition is near chance. Journal of Experimental Psychology: Learning, Memory, and Cognition, 19, 148-155.

Nelson, D. L. (1979). Remembering pictures and words: Appearance, significance, and name. Em L. S. Cermak \& F. I. M. Craik (Orgs.), Levels of processing in human memory (pp. 4576). Hilsdale: Lawrence Erlbaum.

Nelson, T. O. (1971). Savings and forgetting from long-term memory. Journal of Verbal Learning and Verbal Behavior, 10, $568-576$.

Paivio, A. (1971). Imagery and verbal processes. New York: Holt, Rinehart \& Winston.

Parkin, A. J., \& Walter, B. (1992). Recollective experience, normal aging, and frontal dysfunction. Psychology and Aging, 7, 290-298.

Pasquali, L., Veiga, H., Alves, S., \& Vasconcelos, T. (2004). Teste de Memória Visual (TMV). Brasília: LabPam.

Prieto, G., \& Velasco, A. D. (2006). Visualização espacial, raciocínio indutivo e rendimento acadêmico em desenho técnico. Psicologia Escolar e Educacional, 10, 11-20.

Rajaram, S., \& Roediger, H. L. (1997). Remembering and knowing as states of consciousness during recollection. Em J. D. Cohen \& J. W. Scooler (Orgs.), Scientific approaches to the question of consciousness (pp. 213-240). Hillsdale: Erlbaum.

Resolução n. 002. (2003, 24 de março). Define e regulamenta o uso, a elaboração e a comercialização de testes psicológicos e revoga a Resolução CFP n ${ }^{\circ}$ 025/2001. Conselho Federal de Psicologia. Retirado em 15 de dezembro de 2009, de http://site.cfp.org.br/wp-content/uploads/2003/03/resolucao20 03_02_Anexo.pdf

Rueda, F. J. M., \& Sisto, F. F. (2007). Teste Pictórico de Memória (TEPIC-M). Manual. São Paulo: Vetor.

Schacter, D. L., \& Wagner, A. D. (1999). Medial temporal lobe activations in fMRI and PET studies of episodic encoding and retrieval, Hippocampus, 9, 7-24.

Schloerscheidt, A. M., \& Rugg, M. D. (2004). The impact of change in stimulus format on the electrophysiological indices of recognition. Neuropsychologia, 4, 451-466.

Stenberg, G., Radeborg, K., \& Hedman, L. R. (1995). The picture superiority effect in a cross-format recognition task. Memory and Cognition, 23, 425-441.

Tonglet, E. C. (2000). Bateria de Funções Mentais para Motorista (BFM-2) - Teste de memória. São Paulo: Vetor. 
Tonglet, E. C. (2007). Bateria Geral de Funções Mentais (BGFM-4) - Testes de memória de reconhecimento. São Paulo: Vetor.

Tulving, E. (1985). Memory and consciousness. Canadian Psychologist, 21, 1-12.

Tulving, E., \& Craik, F. I. M. (2000). The Oxford handbook of memory. Oxford: Oxford University Press.

Wagner, A. D., \& Gabrieli, J. D. E. (1998). On the relationship between recognition familiarity and perceptual fuency: Evidence for distinct mnemonic processes. Acta Psychologica, 98, 211-230.

Wheeler, M., Stuss, D. T., \& Tulving, E. (1997). Toward a theory of episodic memory: The frontal lobes and autonoetic consciousness. Psychological Bulletin, 121, 331-354.
Yonelinas, A. P., Kroll, N. E. A., Dobbins, I., Lazzara, M., \& Knight, T. (1998). Recollection and familiarity deficits in amnesia: Convergence of remember-know, process dissociation, and receiver operating characteristic data. Neuropsychology, $12,323-339$.
Recebido: 18/02/2010 Última Revisão: 16/01/2012 Aceito: 07/02/2012 